\title{
POLIMORFISMO GHRD3 DEL GEN DEL RECEPTOR DE LA HORMONA DE CRECIMIENTO EN NIÑOS PERUANOS CON TALLA BAJA IDIOPÁTICA
}

\author{
Carlos Del Águila ${ }^{1,2, a}$, César Ortiz²,b, Mirtha Yarlequée,c, Candy Bellido²,b, Miguel Zaldivar ${ }^{2, \mathrm{~d}}$, Juan Falen ${ }^{1, a}$
}

\begin{abstract}
RESUMEN
Objetivos. Describir la estandarización de la detección molecular y la frecuencia del polimorfismo de GHRd3 del gen del receptor de la hormona de crecimiento en una población de niños peruanos con talla baja idiopática. Materiales $y$ métodos. Se estudiaron 64 muestras de sangre periférica de pacientes con diagnóstico de talla baja idiopática atendidos en el servicio de endocrinología del Instituto Nacional de Salud del Niño en Lima, Perú. La amplificación del exón 3 se llevó a cabo empleando los cebadores G1, G2 y G3 optimizándose las condiciones de PCR, como la temperatura de hibridización y las concentraciones de magnesio. Resultados. Se determinó la especificidad de los cebadores a $67{ }^{\circ} \mathrm{C}$ y no se obtuvo diferencias entre las concentraciones de magnesio probadas. El $67 \%$ de los pacientes fueron homocigotos para GHRfl, $28 \%$ fueron heterocigotos y $5 \%$ fueron homocigotos para GHRd3. Conclusiones. La técnica permitió establecer los genotipos de los pacientes con talla baja idiopática, determinándose que solo el $5 \%$ de ellos presenta el genotipo susceptible de mejor respuesta al tratamiento con rhGH, lo cual puede ser considerado en la decisión de iniciar la terapia.
\end{abstract}

Palabras clave: Receptores de hormona del crecimiento; Polimorfismo; Genotipo; Estatura (fuente: DeCS BIREME).

\section{GHRD3 POLYMORPHISM OF GROWTH HORMONE RECEPTOR GEN IN PERUVIAN CHILDREN WITH IDIOPATHIC SHORT STATURE}

\begin{abstract}
Objectives. To describe the standardization of molecular detection and frequency of a growth hormone receptor gene deleted for exon three (GHRd3) polymorphism in a population of Peruvian children with idiopathic short stature. Materials and methods. Peripheral blood samples were used from patients $(\mathrm{N}=64)$ who were diagnosed with idiopathic short stature and were treated at the endocrinology unit of the National Institute of Child Health in Peru The amplification of exon 3 was carried out using G1, G2, and G3 primers by optimizing PCR conditions, such as annealing temperature and magnesium concentration. Results. The specificity of primers was maximized at $67^{\circ} \mathrm{C}$ and there were no differences between magnesium concentration tests. Two-thirds $(67 \%)$ of patients were $\mathrm{GHRfl}$ homozygous, $28 \%$ were heterozygous, and $5 \%$ were GHRd3 homozygous. Conclusions. The test was useful in determining the genotypes of patients with idiopathic short stature and revealed that only $5 \%$ had a genotype that would respond better to rhGH treatment. Thus, molecular assays may be useful when considering the decision to start drug therapy.
\end{abstract}

Key words: Growth hormone receptors; Polymorphism; Genotype; Body height (source: MeSH NLM).

\section{INTRODUCCIÓN}

El receptor de la hormona de crecimiento (GHR) activa vías de transducción de señales que pueden ejercer una acción directa sobre el metabolismo celular, modificando proteínas del citoplasma o, indirectamente, activando factores de transcripción. Un evento bien caracterizado es la producción del factor de crecimiento semejante a la insulina tipo I (IGF-I), el cual favorece la proliferación celular e inhibición de la apoptosis ${ }^{(1-3)}$.

En el humano, el gen GHR está codificado por nueve exones, de los cuales del 3 al 7 codifican el dominio extracelular, cuya función es unirse a la hormona de crecimiento ${ }^{(4)}$. Respecto a este dominio, se han descrito dos isoformas importantes: GHRfl, donde el dominio extracelular está codificado por los exones del 3 al 7 , y $G H R d 3$, donde se ha delecionado el exón $3(2,5)$. Estudios sugieren que el polimorfismo GHRd3 podría ser causa de variabilidad de respuesta al tratamiento con hormona de crecimiento humana recombinante (rhGH) en pacientes con talla baja, aunque este hecho no está totalmente esclarecido ${ }^{(6,7)}$.

La diferencia de respuesta a la terapia observada en pacientes con talla baja idiopática que reciben $\mathrm{rhGH}$,

\footnotetext{
Hospital Nacional de Salud del Niño. Lima, Perú.

Facultad de Medicina, Universidad Nacional Federico Villarreal. Lima, Perú.

a Médico pediatra endocrinólogo; ${ }^{\mathrm{b}}$ biólogo, genetista biotecnólogo; ${ }^{\mathrm{b}}$ biólogo magíster en Recursos Vegetales y Terapéuticos; ${ }^{\mathrm{b}}$ biólogo, magíster en Bioquímica.

Recibido: 09-06-15 Aprobado: 27-11-15
}

Citar como: Del Águila C, Ortiz C, Yarlequé M, Bellido C, Zaldivar M, Falen J. Polimorfismo GHRD3 del gen del receptor de la hormona de crecimiento en niños peruanos con talla baja idiopática. Rev Peru Med Exp Salud Publica. 2016;33(1):45-50. doi: 10.17843/rpmesp.2016.331.1898 
puede deberse a factores como la edad, talla de inicio, dosis y duración del tratamiento, así como retardo en la edad ósea; no obstante, los factores genéticos también pueden estar involucrados en dicha variabilidad, y es donde el estudio del polimorfismo GHRd3 puede tener impacto ${ }^{(6,7)}$. El efecto del polimorfismo GHRd3 ha sido estudiado en diferentes enfermedades que afectan el crecimiento, incluyendo niños con talla baja idiopática, demostrándose que pacientes con uno o dos alelos GHRd3 presentan una velocidad de crecimiento mayor en el primer y segundo año de tratamiento con $\mathrm{rhGH}$, comparado con aquellos homocigotos para GHRfl (8-11). Si bien existen estudios que indican que la afinidad del receptor GHRd3 por la $\mathrm{GH}$ no se ve afectada, otros estudios sugieren que la deleción del exón 3 puede cumplir una función fundamental en los cambios conformacionales durante la transactivación de los dímeros de GHR por parte de la hormona de crecimiento $(8,12)$. Estos estudios dan cuenta de la importancia de individualizar la terapia según el genotipo del paciente, lo que resalta aun más, considerando el gasto económico elevado que implica el tratamiento, además de no ser de fácil acceso en Perú.

En el presente estudio se describe la estandarización de la detección molecular y la frecuencia del polimorfismo GHRd3 en una población de niños con talla baja idiopática, atendidos en el Instituto Nacional de Salud del Niño (INSN) en Lima, Perú.

\section{MATERIALES Y MÉTODOS}

\section{MUESTRAS ANALIZADAS}

Estudio descriptivo donde se evalúa 64 pacientes con talla baja idiopática, referidos al Servicio de Endocrinología del INSN, entre enero de 2013 a enero de 2015. Los pacientes cumplían con los criterios de consenso para talla baja idiopática, que considera aquella talla/edad por debajo de 2 desviaciones estándar de la mediana según edad y en ausencia de alguna enfermedad metabólica, endocrina, $u$ otra que explique la talla baja ${ }^{(13)}$. Se utilizaron pruebas de estimulación farmacológicas a la hormona de crecimiento con clonidina y L. dopa que resultaron con valores normales. Se usó el método por RIA (radioinmmune assay) y se consideró normal una concentración de hormona de crecimiento mayor a $7 \mathrm{ng} / \mathrm{dL}$. El pico de $\mathrm{GH}$ promedio fue de $12,9 \pm 2,1 \mathrm{ng} / \mathrm{mL}$. Para el análisis molecular las muestras fueron enviadas al Laboratorio de Bioquímica y Biología Molecular de la Facultad de Medicina de la Universidad Nacional Federico Villarreal.

\section{EXTRACCIÓN DE ADN}

El ADN fue extraído a partir de 52 muestras de sangre periférica en tubos con EDTA (etilendiaminotetracético) y 12 muestras de sangre seca en tarjetas FTA ${ }^{\mathrm{TM}}$. En el primer caso el ADN se extrajo a partir de un kit de extracción de ácidos nucleicos (Roche), empleando $200 \mu \mathrm{L}$ de sangre periférica incubados con buffer de lisis y proteinasa K. Los lisados fueron cargados a tubos con filtro de sílica en el cual el ADN fue lavado y eluído para su posterior análisis. EI ADN de sangre seca en tarjetas FTA ${ }^{\mathrm{TM}}$ fue purificado a partir de discos de $2 \mathrm{~mm}$ de diámetro sometidos a lavados sucesivos con reactivos de purificación (GE Healthcare). Los discos lavados fueron empleados como muestras en los protocolos de PCR.

\section{ESTANDARIZACIÓN DE LA REACCIÓN EN CADENA DE LA POLIMERASA (PCR)}

La PCR se realizó usando los cebadores propuestos por Pantel et al. (6) G1: 5'-TGTGCTGGTCTGTTGGTCTG-3'; G2: 5'AGTCGTTCCTGGGACAGAGA-3'; y G3: 5'-CCTGGATTAACACTTTGCAGACTC-3' (Figura 1). Para la estandarización de la PCR se probaron diferentes concentraciones de ión $\mathrm{Mg}^{++}$en el rango de $1,5 \mathrm{mM}$ a $3 \mathrm{mM}$, y diferentes temperaturas de hibridización de los cebadores en el rango de 60 a $67^{\circ} \mathrm{C}$. Las concentraciones de cebadores se fijaron en $0,8 \mu \mathrm{M}$, las de dNTPs en $250 \mu \mathrm{M}$ y la de Taq polimerasa en 0,5 U/ $\mu \mathrm{L}$. Las condiciones de PCR fueron 2 min a $94{ }^{\circ} \mathrm{C}$, seguidos de 35 ciclos de $94^{\circ} \mathrm{C}$ por $30 \mathrm{~s}, 60-67^{\circ} \mathrm{C}$ por $30 \mathrm{~s}$, y $72^{\circ} \mathrm{C}$ por $1 \mathrm{~min}$ $20 \mathrm{~s}$; luego, una etapa de extensión final a $72{ }^{\circ} \mathrm{C}$ por $5 \mathrm{~min}$. Los productos de amplificación se visualizaron por electroforesis en geles de agarosa al $2 \%$, teñidos con RunSafe (Cleaver Scientific).

\section{GENOTIPIFICACIÓN GHRFL/GHRD3}

El análisis de los genotipos se realizó mediante dos reacciones de PCR, una múltiplex con los cebadores $\mathrm{G} 1$, G2 y G3 para la detección simultánea de los dos alelos GHRd3 y GHRfl (532 pb y 935 pb, respectivamente) (Figura 1), y una reacción monoplex con los cebadores G1 y G3 para confirmar el genotipo GHRfl homocigoto, según recomendación de Álvarez-Nava et al. (14). Los resultados fueron analizados en geles de agarosa al $2 \%$, teñidos con RunSafe (Cleaver Scientific). Los pacientes fueron clasificados como genotipo homocigoto GHRd3 y homocigoto GHRfl si se observó la amplificación de un único alelo con una banda de 532 o 935 pb en la electroforesis, respectivamente, y como heterocigoto GHRfl/GHRd3 si se observaron las dos bandas. Se calculó el equilibrio de Hardy-Weinberg de acuerdo con el procedimiento estándar utilizando el análisis de chi cuadrado.

\section{CONSIDERACIONES ÉTICAS}

Por cada paciente se utilizaron muestras de sangre periférica obtenidas bajo consentimiento informado. El protocolo del estudio fue aprobado por el Comité de Ética del INSN. 


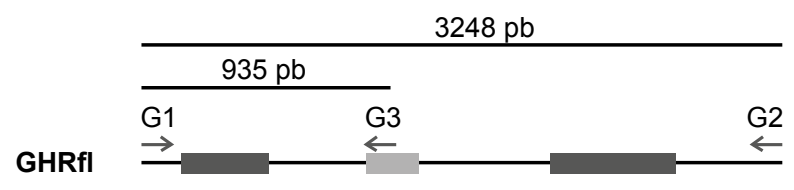

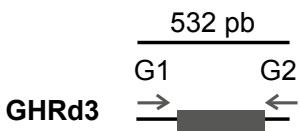

Figura 1. Estrategia de detección de GHRfl y GHRd3. Los cebadores $G 1$ y $G 3$ permiten la detección del alelo GHRfl mediante una banda de $935 \mathrm{pb}$. G3 es el cebador que realiza la amplificación diferencial hibridando en la región del exón 3 . El fragmento de 3248 pb no es amplificado bajo las condiciones empleadas. Los cebadores $\mathrm{G} 1$ y $\mathrm{G} 2$ detectan del alelo $\mathrm{GHRd} 3$ amplificando un fragmento de $532 \mathrm{pb}$.

\section{RESULTADOS}

\section{DESCRIPCIÓN DEL GRUPO DE ESTUDIO}

Se estudiaron 42 niños de sexo masculino y 22 de sexo femenino con un puntaje $Z$ talla/edad promedio de $-2,7$ $\pm 0,3$ e índice de masa corporal de 15,9 $\pm 0,5$. La edad promedio fue de $8,9 \pm 0,5$.

\section{ESTANDARIZACIÓN DE LAPCR}

No se observaron diferencias en la eficiencia de la PCR al probar distintas concentraciones de ión $\mathrm{Mg}++$ (datos no mostrados), por lo que se estableció arbitrariamente la concentración de $2,5 \mathrm{mM}$ para realizar los análisis subsiguientes. La temperatura de hibridización óptima fue de $67^{\circ} \mathrm{C}$, siendo la única temperatura en la cual en la PCR fue específica, es decir, a dicha temperatura, los cebadores se unen a su secuencia complementaria exacta y no a secuencias solo similares, por lo que no generan bandas de tamaño diferentes a las esperadas en la electroforesis (Figura 2).

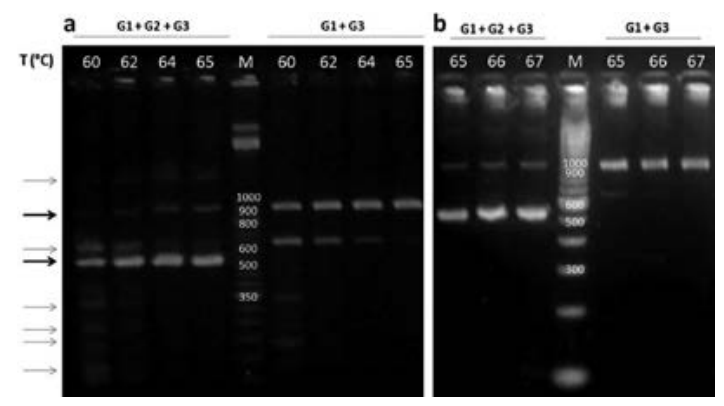

Figura 2. Estandarización de la $P C R$ para la detección de GHRfl y GHRd3. A la izquierda del marcador de peso molecular (M) la reacción multiplex y a la derecha la reacción monoplex. a. Se observa un incremento de la especificidad de la PCR al aumentar la temperatura de hibridización de los cebadores de 60 a $65{ }^{\circ} \mathrm{C}$. $(\rightarrow)$ Indica las bandas inespecíficas que desaparecen con el incremento de la temperatura. $(\rightarrow)$ Indica las bandas que corresponden a los alelos GHRfl (935 pb) y GHRd3 (532 pb). b. Se observa que la reacción se hace totalmente específica a la temperatura de $67^{\circ} \mathrm{C}$. M: marcador de peso molecular $(\mathrm{pb})$

\section{GENOTIPIFICACIÓN GHRfI/GHRd3}

Se halló una elevada frecuencia del genotipo homocigoto GHRfl (67\%) sobre el del homocigoto GHRd3 (5\%). Además, se determinó una frecuencia intermedia de heterocigotos (28\%) (Figura 3b). La genotipificación no fue afectada por el método de obtención de la muestra, sangre periférica líquida en tubos con EDTA o sangre periférica seca en tarjetas $\mathrm{FTA}^{\mathrm{TM}}$. Estas frecuencias cumplen con la Ley de Hardy-Weinberg habiéndose obtenido en el test de $x^{2}$ un valor $p$ de 0,37 .

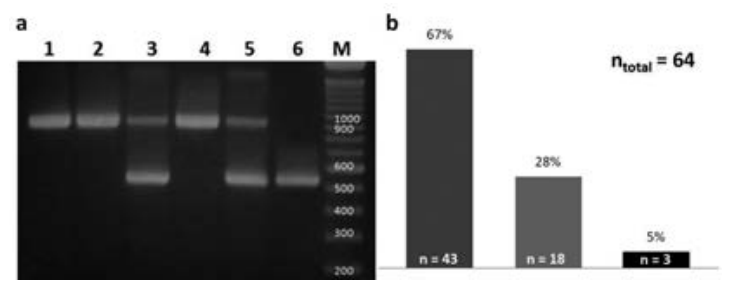

Figura 3. Análisis del polimorfismo del exón 3 del gen GHR. a. Electroforesis en agarosa al $2 \%$ para genotipificación GHRfl/ GHRd3 (se muestra solo la reacción multiplex). La presencia de una única banda de $935 \mathrm{pb}$ indica genotipo homocigoto GHRfl (columnas 1, 2 y 4); una banda de 532 pb indica genotipo homocigoto GHRd3 (columna 6), y la presencia de dos bandas indica el genotipo heterocigoto (columnas 3 y 5 ). M: marcador de peso molecular (pb). b. Frecuencias genotípicas GHRfI/ GHRd3 en pacientes con talla baja idiopática. Se observa que la frecuencia del genotipo GHRd3 homocigoto solo alcanza el $5 \%$ de la población analizada.

\section{DISCUSIÓN}

La activación del receptor de la hormona de crecimiento (GHR) puede verse influenciada por alteraciones genéticas de los exones del gen que la codifica. Godowski et al. ${ }^{(15)}$ fueron los primeros en describir en dos pacientes, las deleciones en los exones 3,5 y 6 , señalando que podrían ser la causa de la insensibilidad al tratamiento con la hormona de crecimiento observada en dichos pacientes. En la actualidad se reconocen dos isoformas con implicancia clínica: una en su total longitud (GHRfl) y otra con el exón 3 delecionado (GHRd3). La isoforma GHRd3 se habría originado en el proceso de recombinación homóloga de dos secuencias retrovirales que flanquean el exón 3 en el hombre ${ }^{(6)}$.

La detección molecular de la variante polimórfica GHRd3 se realiza en dos momentos; en un primer momento se lleva a cabo mediante PCR múltiplex, usando tres cebadores (G1, G2 y G3), de los cuales el G3 permite la detección del alelo GHRfl y el G2 del alelo GHRd3 (6). En los casos que resulten heterocigotos u homocigotos para GHRfl, el análisis culmina; sin embargo, los casos que resulten homocigotos para $G H R d 3$ requieren un segundo 
análisis confirmatorio, el cual consiste en realizar una PCR convencional monoplex, usando los cebadores $\mathrm{G} 1$ y G3, para la detección solo del alelo GHRfl. En el presente estudio, un caso se halló inicialmente como homocigoto para GHRd3 y resultó ser heterocigoto en el segundo análisis. Lo que quiere decir que la PCR múltiplex puede conducir a obtener falsos homocigotos GHRd3. Ello se debería a una competencia entre la amplificación de los alelos GHRfl y GHRd3 en la PCR múltiplex, en la que el fragmento de menor tamaño (GHRd3 de $532 \mathrm{pb}$ ) ve favorecida su amplificación (Figura 3a). Respecto a los métodos de obtención de la muestra, no evidenciamos problemas en el procedimiento técnico entre usar sangre periférica líquida en tubos con EDTA o sangre periférica seca en tarjetas FTA $^{\mathrm{TM}}$, ni observamos ninguna influencia en el resultado de la genotipificación. El uso de tarjetas FTA $^{\mathrm{TM}}$ podría resultar una ventaja al trabajar con población pediátrica, aunque debe mencionarse que por el volumen de sangre obtenido por este método, la cantidad de análisis genéticos posibles son menores respecto al empleo de sangre líquida.

La importancia del estudio del polimorfismo GHRd3 radica en su implicancia en el tratamiento que reciben los pacientes con problemas de talla baja. En el año 2001 y 2003 la FDA aprobó el uso de la hormona de crecimiento recombinante humana ( $r \mathrm{hH}$ ) en niños pequeños para la edad gestacional (SGA) y en niños con talla baja idiopática, respectivamente ${ }^{(8)}$. Los estudios indican que las personas que tienen el alelo GHRd3 responden mejor a la rhGH con respecto a aquellos que poseen el alelo GHRfl ${ }^{(15)}$. La explicación es aún motivo de controversia, aunque existe la hipótesis de que GHRd3 favorece la actividad del receptor por cambios conformacionales en el dominio extracelular en respuesta a su unión con la hormona de crecimiento ${ }^{\left({ }^{8}\right)}$. Si se tienen en consideración que los residuos de aminoácidos codificados por el exón 3 no se encuentran localizados en la superficie de unión a la hormona de crecimiento, las diferencias de afinidad del receptor por su ligando, no es una explicación convincente para explicar las diferencias biológicas entre ambas isoformas ${ }^{(16)}$.
El admitir que el polimorfismo GHRd3 sería un marcador de respuesta a la terapia es una explicación plausible; sin embargo, aún no se ha logrado consenso sobre su utilidad. Los estudios de la respuesta a la rhGH en pacientes con talla baja con presencia o no de GHRd3 tienen como fundamento la medida de la velocidad de la talla de los pacientes durante el primer y el segundo año de tratamiento. De otro lado, debe señalarse que estos estudios han involucrado diversos tipos de condiciones como: pequeños para la edad gestacional, talla baja idiopática, síndrome de Turner y deficiencia de hormona de crecimiento (10); habiéndose obtenido datos contradictorios, aunque en muchos estudios se ha encontrado la existencia de asociación del polimorfismo GHRd3 con incremento de mayor velocidad de crecimiento $\mathrm{y}$, por lo tanto, de la talla del paciente que recibe $\mathrm{rhGH}^{(9-12)}$. Así, Jorge et al. ${ }^{(10)}$, señalan que se deben tener en cuenta criterios de inclusión adecuados para un buen análisis. Dichos autores estudiaron pacientes con deficiencia de hormona de crecimiento, encontrando asociación entre la respuesta a rhGH y $\mathrm{GHRd3}$, e indicaron que los estudios contradictorios se debían a la inclusión de sujetos que tenían niveles hormonales inferiores a $10 \mathrm{ng} / \mathrm{mL}$, que luego de la prueba de estudio de reserva pituitaria de $\mathrm{GH}$ se comportaran como no deficientes, proponiendo el uso del corte de $7 \mathrm{ng} / \mathrm{mL}$ cuando la hormona es medida mediante RIA y $3,3 \mathrm{ng} / \mathrm{mL}$ cuando se utilizaba la inmunofluorometría monoclonal.

Una vez establecida la importancia y utilidad del GHRd3, debe tenerse en cuenta la distribución alélica y genotípica del polimorfismo en la población, lo que da la idea de cuantos pacientes en algún momento podrían beneficiarse de su detección. Los reportes indican que el alelo GHRd3 está distribuido en promedio en el $15 \%$ de la población general, encontrando la frecuencia del genotipo homocigoto GHRd3 entre el 5 y al $20 \%{ }^{(6,7,14,17-24)}$. A la fecha, no existe un reporte del mismo en población peruana. El presente estudio no tiene un enfoque poblacional, sin embargo, llama la atención la baja frecuencia del genotipo homocigoto para GHRd3 (5\%) (Figura 3) (Tabla 1).

Tabla 1. Distribución de las frecuencias genotípicas del polimorfismo GHRd3 en diferentes poblaciones del mundo

\begin{tabular}{|c|c|c|c|c|c|}
\hline Referencia & País & $\mathbf{n}$ & GHRfI/GHRfI (\%) & GHRfl/GHRd3 (\%) & GHRd3/GHRd3 (\%) \\
\hline (6) & Francia & 150 & 58 & 33 & 9 \\
\hline (7) & España & 289 & 27 & 57,8 & 15,2 \\
\hline (14) & Venezuela & 50 & 56 & 30 & 14 \\
\hline (17) & Turquía & 477 & 35 & 39 & 26 \\
\hline (18) & Alemania & 62 & 45 & 40 & 15 \\
\hline (19) & Canadá & 368 & 53,3 & 35,6 & 11,1 \\
\hline (20) & Suiza & 211 & 47,9 & 43,6 & 8,5 \\
\hline (21) & Benin & 154 & 30 & 44 & 26 \\
\hline (22) & Inglaterra & 100 & 53 & 40 & 7 \\
\hline (23) & Brasil & 105 & 54,3 & 36,2 & 9,5 \\
\hline (24) & Argentina & 192 & 60 & 34 & 6 \\
\hline Presente estudio & Perú & $64^{*}$ & 67 & 28 & 5 \\
\hline
\end{tabular}

* La población peruana corresponde a pacientes con talla baja idiopática, a diferencia del resto de estudios indicados donde se presenta a una población testigo 
El presente estudio posee limitaciones. Este reporte está orientado a dar a conocer la importancia del polimorfismo GHRd3 en pacientes candidatos a recibir terapia con rhGH, y describir la detección molecular del mismo. Queda pendiente la inclusión de pacientes bajo terapia con rhGH y la correlación de la respuesta al tratamiento con el polimorfismo GHRd3. Además, los pacientes aquí estudiados padecen todos de talla baja idiopática, dicha característica no requiere ninguna conclusión de momento, por lo menos no hasta incluir una población testigo, con la cual compararla. Finalmente, es de suma importancia considerar el estudio de la distribución de GHRd3 en población peruana. Los resultados aquí presentados indican que la frecuencia de $G H R d 3$ es baja y probablemente similar a lo reportado a otras poblaciones.

Finalmente, el polimorfismo GHRd3 es un factor genético que modula la respuesta a la terapia con $\mathrm{hGH}$, lo cual ha sido estudiado en diversas alteraciones del crecimiento. La detección molecular permite su identificación rápida proveyendo al clínico una herramienta más en la decisión del tratamiento adecuado para el paciente.

Agradecimientos: al biólogo Dan Vivas, investigador de la Universidad Nacional Mayor de San Marcos y a la bióloga Mercedes Palomino, por la colaboración prestada en la implementación de la metodología. Al Instituto Nacional de Salud del Niño y a la Facultad de Medicina Hipólito Unanue de la Universidad Nacional Federico Villarreal por el apoyo económico que hizo posible la ejecución del Proyecto.

Contribuciones de autoría: CDA, CO, MY y MZ han participado en la concepción y diseño del trabajo, análisis e interpretación de datos, revisión crítica del manuscrito y aprobación de su versión final. OC y BC participaron en la obtención de los resultados, y CDA, MY, MZ y FJ colaboraron en la revisión crítica del artículo y aprobación de su versión final.

Fuentes de financiamiento: el presente trabajo fue financiado por el Instituto Nacional de Salud del Niño y por la Facultad de Medicina de la Universidad Nacional Federico Villarreal.

Conflictos de interés: los autores declaran no tener conflictos de interés en la publicación del presente trabajo.

\section{REFERENCIAS BIBLIOGRÁFICAS}

1. Herrington J, Carter-Su C. Signaling pathways activated by the growth hormone receptor. Trends Endocrinol Metab. 2001;12(6):252-7

2. Pantel J, Grulich-Henn J, Bettendorf $M$, Strasburger CJ, Heinrich U, Amselem S. Heterozygous Nonsense Mutation in Exon 3 of the Growth Hormone Receptor $(G H R)$ in Severe GH Insensitivity (Laron Syndrome) and the Issue of the Origin and Function of the GHRd3 Isoform. J Clin Endocrinol Metab. 2003;88(4):1705-10.

3. Filopanti M, Giavoli C, Grottoli S, Bianchi A, De Marinis L, Ghigo $\mathrm{E}$ et al. The exon 3-deleted growth hormone receptor: Molecular and functional characterization and impact on GH/IGF-I axis in physiological and pathological conditions. J Endocrinol Invest. 2011;34(11):861-8

4. Rodriguez S, Gaunt TR, Day INM. Molecular genetics of human growth hormone, insulin-like growth factors and their pathways in common disease. Hum Genet. 2007;122(1):1-21.

5. Yang N, Langenheim JF, Wang $\mathrm{X}$, Jiang J, Chen WY, Frank SJ. Activation of Growth Hormone Receptors by Growth Hormone and Growth Hormone Antagonist Dimers: Insights into Receptor Triggering. Mol Endocrinol. 2008;22(4):978-88.
6. Pantel J, Machinis K, Sobrier ML, Duquesnoy P, Goossens M, Amselem S. Species-specific alternative splice mimicry at the growth hormone receptor locus revealed by the lineage of retroelements during primate evolution. J Biol Chem. 2000;275(25):18664-9.

7. Audi L, Esteban C, Carrascosa A, Espadero R, Perez-Arroyo A, Arjona R, et al. Exon 3-deleted/fulllength growth hormone receptor polymorphism genotype frequencies in Spanish short small-for-gestationalage (SGA) children and adolescent $(n=247)$ and in an adult control population $(n=289)$ show increased $\mathrm{A} / \mathrm{A}$ in short SGA. J Clin Endocrinol Metab. 2006;91(12):5038-43.

8. Dos Santos C, Essioux L, Teinturier C, Tauber M, Goffin V, Bougnères P. A common polymorphism of the growth hormone receptor is associated with increased responsiveness to growth hormone. Nat Genet. 2004;36(7):720-4.

9. Bakker B, Frane J, Anhalt H, Lippe B, Rosenfeld RG. Height velocity targets from the national cooperative growth study for first-year growth hormone responses in short children. J Clin Endocrinol Metab. 2008;93(2):352-7.

10. Jorge AA, Marchisotti FG, Montenegro LR, Carvalho LR, Mendonca BB,
Arnhold IJ. Growth Hormone (GH) Pharmacogenetics: Influence of $\mathrm{GH}$ Receptor Exon 3 Retention or Deletion on First-Year Growth Response and Final Height in Patients with Severe GH Deficiency. J Clin Endocrinol Metab. 2006;91(3):1076-80.

11. Blum WF, Machinis K, Shavrikova EP, Keller A, Stobbe H, Pfaeffle $\mathrm{RW}$, et al. The growth response to growth hormone $(\mathrm{GH})$ treatment in children with isolated $\mathrm{GH}$ deficiency is independent of the presence of the exon 3-minus isoform of the $\mathrm{GH}$ receptor. J Clin Endocrinol Metab. 2006;91(10):4171-4.

12. Pilotta A, Mella P, Filisetti M, Felappi B, Prandi E, Parrinello G, et al. Common Polymorphisms of the Growth Hormone (GH) Receptor Do Not Correlate with the Growth Response to Exogenous Recombinant Human GH in GHDeficient Children. J Clin Endocrinol Metab. 2006;91(3):1178-80.

13. Cohen P, Rogol AD, Deal CL, Saenger P, Reiter EO, Ross JL, Chernausek SD, Savage MO, Wit JM, 2007 ISS Consensus Workshop participants. Consensus statement on the diagnosis and treatment of children with idiopathic short stature: a summary of the Growth Hormone Research 
Society, the Lawson Wilkins Pediatric Endocrine Society, and the European Society for Paediatric Endocrinology Workshop. J Clin Endocrinol Metab. 2008;93(11):4210-7. doi: 10.1210/ jc.2008-0509.

14. Alvarez-Nava F, Lanes R, Marcano H, Pardo T, Zabala W, Quintero JM et al. Distribución de las frecuencias alélicas y genotípicas del polimorfismo GHRd3 en pacientes venezolanos con talla baja. Rev Venez Endocrinol Metab. 2009:7(1):26-34.

15. Godowski PJ, Leung DW, Meacham LR, Galgani JP, Hellmiss R, et al. Characterization of the human growth hormone receptor gene and demonstration of a partial gene deletion in two patients with larontype dwarfism. Proc Natl Acad Sci U S A. 1989;86(20):8083-7.

16. Jorge AA, Arnhold IJ. Growth Hormone Receptor Exon 3 Isoforms and Their Implication in Growth Disorders and Treatment. Horm Res. 2009;71 Suppl 2:55-63. doi: $10.1159 / 000192438$.

17. Bas F, Kelesoglu F, Timirci O, Kabatas S, Bozkurt N, Kucukemre B, et al. The Distribution of Exon 3-Deleted/ Full-Length Growth Hormone Receptor Polymorphism in the Turkish Population. J Clin Res Ped Endo. 2011;3(3):126-31. doi: 10.4274/jcrpe. v3i3.25.
18. Binder G, Baur F, Schweizer R, Ranke $\mathrm{MB}$. The d3-growth hormone $(\mathrm{GH})$ receptor polymorphism is associated with increased responsiveness to $\mathrm{GH}$ in Turner syndrome and shortfor-gestational-age children. J Clin Endocrinol Metab. 2006;91(2):659-64.

19. Kenth G, Shao Z, Cole DEC, Goodyer CG. Relationship of the human growth hormone receptor exon 3 genotype with final adult height and bone mineral density. J Clin Endocrinol Metab. 2007;92(2):725-8.

20. Raz B, Janner M, Petkovic V, Lochmatter D, Eble A, Dattani T et al. Influence of growth hormone (GH) receptor deletion of exon 3 and fulllenght isoforms on $\mathrm{GH}$ response and final height in patients with severe $\mathrm{GH}$ deficiency. J Clin Endocrinol Metab. 2008;93(3):974-80.

21. Millar DS, Lewis MD, Horan M, Newsway V, Rees DA, Easter TE et al. Growth hormone (GH1) gene variation and the growth hormone receptor (GHR) exon 3 deletion polymorphism in a West-African population. Mol Cell Endocrinol. 2008;296(1-2):18-25. doi: 10.1016/j. mce.2008.09.023.

22. Adetunji OR, MacFarlane IA, Javadpour M, Alfirevic A, Pirmohamed M, Blair JC. The d3/fl-GH receptor gene polymorphism does not influence quality of life and body composition in $\mathrm{GH}$-deficient adults receiving $\mathrm{GH}$ replacement therapy. Eur J Endocrinol. 2009;161(4):541-6. doi: 10.1530/ EJE-09-0405.

23. Marques FA, Lins TC, Lima RM, Fonseca RM, de Franca NM, de Oliveira $\mathrm{RJ}$ et al. The exon 3 polymorphism of the growth hormone receptor is a severity-related factor for osteoporosis. Endocrine. 2014;45(3):487-96. doi: 10.1007/s12020-013-0004-1.

24. Ballerini MG, Domené HM, Scaglia P, Martinez A, Keselman A, Jasper HG et al. Association of serum components of the GH-IGFs-IGFBPs system with GHR-exon 3 polymorphism in normal and idiopathic short stature children. Growth Horm IGF Res. 2013;23(6):229-36. doi: 10.1016/j. ghir.2013.08.003.

Correspondencia: Carlos Del Águila Villar. Dirección: Avenida Santa Cruz, 647, Miraflores. Lima, Perú.

Teléfono: (511) 999653731

Correo electrónico: caguilav@hotmail.com

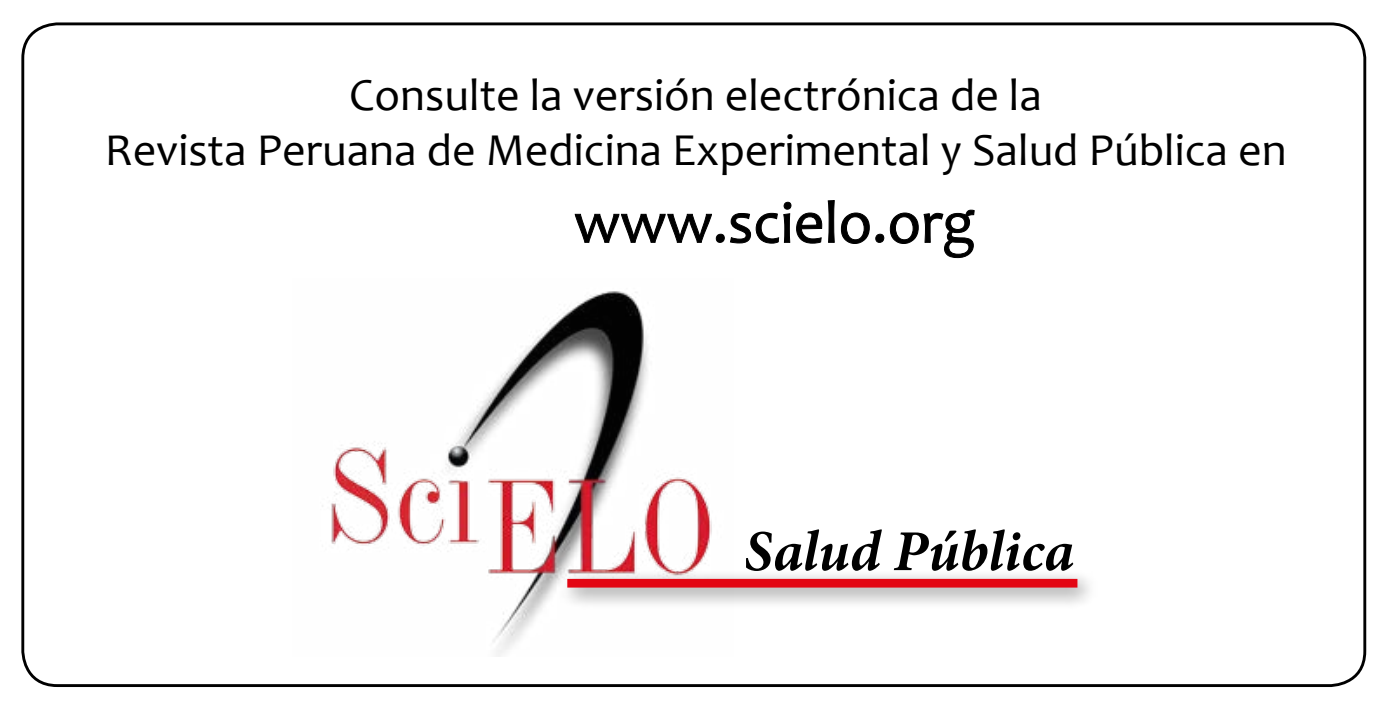

